\title{
Prevalencia de factores de riesgo cardiovascular clásicos en población adulta de Talca, Chile, 2005
}

\author{
Iván Palomo G 1,a, G loria Icaza N 2,b, Verónica Mujica E ${ }^{1,4}$, \\ Loreto $N$ úñez $F^{3, c}$, Elba Leiva $M^{1, d}$, Marcela Vásquez $R^{1, d}$, \\ Marcelo Alarcón $L^{1, f}$, Emilio Moyano $D^{5, e}$. \\ Prevalence of cardiovascular risk \\ factors in adult from Talca, Chile
}

Background: Cardiovascular disease (CVD) has several traditional risk factors $(\mathrm{RF})$, and some of them are potentially modifiable. Aim: To determine the prevalence of most common risk factors in adult population in Talca in Central Chile. Subjects and methods: We studied 1007 subjects aged 18 to 74 years (66\% women), living in Talca, selected by a probability sampling. They answered a questionnaire and anthropometry, blood pressure, lipid profile and blood glucose were measured. Results: Thirty seven percent of subjects smoked, $70.1 \%$ had a body mass index over $25 \mathrm{~kg} / \mathrm{m}^{2}$ and $41 \%$ had an abnormally high circumference. High blood pressure was found in 37\% (36\% of these subjects were unaware of this condition), $44.5 \%$ had hypercholesterolemia, $21.5 \%$ had low HDL cholesterol, $40.1 \%$ had hypertriglyceridemia and $26.3 \%$ had high blood glucose levels. Conclusions: There is a high prevalence of risk factors for CVD in adult population from Talca. The prevalence of risk factors is higher than that reported by the 2003 National Health Survery (Rev Méd Chile 2007; 135: 904-12).

(Key words: Cardiovascular diseases; Obesity; Overweight; Risk factors)

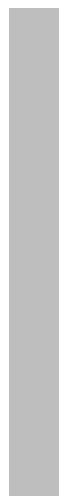

Recibido el 11 de octubre, 2006. Aceptado el 4 de enero, 2007.

Proyecto realizado por el Programa de Investigación en Factores de Riesgo de Enfermedades Cardiovasculares de la Universidad de Talca.

${ }_{1}^{1}$ Departamento de Bioquímica Clínica e Inmunohematología, Facultad de Ciencias de la Salud, Universidad de Talca, Talca, Chile. ${ }^{2}$ Instituto de Matemática y Física, Universidad de Talca, Talca, Chile. ${ }^{3}$ Departamento de Salud Pública, Facultad de Ciencias de la Salud, Universidad de Talca, Talca, Chile. ${ }^{4}$ Servicio de Medicina, Hospital Regional de Talca, Chile. ${ }^{5}$ Escuela de Psicología, Universidad de Talca, Talca, Chile.

Tecnólogo Médico, PhD

bioestadística, $\mathrm{PhD}$

${ }^{\mathrm{c} O d o n t o ́ l o g o, ~ M g}$

dTecnólogo Médico, Mg

ePsicólogo, PhD

fTecnólogo Médico, Lic

Correspondencia a: Iván Palomo G, PhD. Departamento de Bioquímica Clínica e Inmunohematología, Facultad de Ciencias de la Salud, Universidad de Talca, Talca, Chile. Casilla 747, Talca, Chile. Tel-Fax: 56-71-200488. E mail: ipalomo@utalca.cl 
$\mathrm{D}$ e acuerdo a la Organización Mundial de la Salud, las enfermedades cardiovasculares (ECV), representan alrededor de $30 \%$ de las defunciones mundiales ${ }^{1}$. En Chile, producto de la transición demográfica y epidemiológica de las últimas décadas, la situación es similar ${ }^{2,3}$. Recientemente Icaza y Núnez ${ }^{4}$ mostraron que en la comuna de Talca, durante el período 1997-2003, la tasa de mortalidad por enfermedades isquémicas del corazón (98,3/100 mil habitantes) fue similar a la tasa nacional (103,9/100 mil habitantes) y la tasa de mortalidad por enfermedades cerebrovasculares $(115,5 / 100$ mil habitantes) fue significativamente mayor a la tasa nacional $(99,1 / 100$ mil habitantes).

En el contexto de los factores de riesgo cardiovascular (FRCV) clásicos ${ }^{5}$, es conocido que la hipertensión arterial (HTA), hipercolesterolemia, diabetes mellitus (DM) y tabaquismo, son los FRCV mayores y la obesidad y sedentarismo FRCV condicionantes. La Encuesta Nacional de Salud, realizada en 2003 (ENS) por el Ministerio de Salud ${ }^{6}$ mostró una alta prevalencia de FRCV. Dado que la mayoría de los FRCV son modificables, es importante conocer su prevalencia a nivel local, información que será útil a la autoridad sanitaria para diseñar e implementar programas que apunten a disminuir su frecuencia en la población.

\section{SujeTOS Y MÉTODOS}

Población y diseño del estudio. Para medir la prevalencia de FRCV en la población adulta de 18 a 74 años de la ciudad de Talca, se realizó un estudio transversal descriptivo. Las embarazadas y personas con discapacidad mental no fueron incluidas. El tamaño muestral se calculó con un nivel de confianza de 95\% y un error de 3\%, considerando una prevalencia conservadora de $50 \%$. Para compensar las pérdidas de sujetos y controlar por variables confundentes, el tamaño muestral se incrementó en $20 \%$. Se realizó un muestreo probabilístico polietápico, en cuya primera etapa se enumeraron las manzanas por distritos censales, luego mediante muestreo aleatorio simple, se eligieron 361 manzanas de la ciudad. En la segunda etapa, mediante un procedimiento de selección sistemática, se eligieron ocho casas por manzana. Una vez seleccionada la casa, encuestadores entrenados seleccionaron a un sujeto por domicilio usando la tabla de Kish ${ }^{7}$. A las personas elegidas que aceptaron participar en el estudio, se les solicitó firmar un consentimiento informado y contestar un cuestionario.

Recolección de datos. La recolección de la información se realizó entre septiembre y diciembre del año 2005. El cuestionario comprendía preguntas sobre caracterización del entrevistado, nivel socioeconómico (NSE) del hogar, tabaquismo, actividad física y antecedentes mórbidos personales y familiares. Los entrevistados fueron citados a la Universidad de Talca para realizar mediciones antropométricas y extracción de sangre venosa, previo ayuno de 12 a $14 \mathrm{~h}$.

La mediciones de peso, talla y perímetro de cintura se realizaron bajo condiciones estándares ${ }^{8}$. En las mediciones antropométricas se utilizó una balanza calibrada periódicamente y un antropómetro. Las mediciones se realizaron sin zapatos y con ropa mínima. Para la medición de la presión arterial se utilizó un monitor de inflado automático (OMRON HEM-741CINT) y se siguió el procedimiento descrito en la Guía Clínica Examen de Medicina Preventiva ${ }^{9}$.

Para las mediciones de perfil lipídico y glicemia se utilizó reactivos de marca Roche Laboratories, Mannheim, Alemania y un espectrofotómetro automático (Hitachi 717, Japón). El colesterol-LDL fue calculado por la fórmula de Friedewald ${ }^{10}$.

Las variables en estudio se operacionalizaron de la siguiente manera:

a) Características generales. La población fue agrupada en cuatro grupos etarios (18-24, 2544 , 45-64 y 65-74 años), siguiendo las categorías de la $\mathrm{ENS}^{6}$, cinco niveles socioeconómicos (muy alto, alto, medio alto, medio bajo y bajo $)^{11}$ y tres niveles de escolaridad (<8, 8-12 y $>12$ años) al igual que en la ENS 6 .

b) Presión arterial. Se definió como presión arterial alta $\geq 140 \mathrm{mmHg}$ sistólica $0 \geq 90 \mathrm{mmHg}$ diastólica ${ }^{12,13}$. Los sujetos que ingerían antihipertensivos fueron considerados como hipertensos.

c) Perfil lipídico. Se consideró colesterol elevado $>200 \mathrm{mg} / \mathrm{dl}^{14,15}$. El colesterol-HDL se consideró alterado con cifras $<40$ y $<50 \mathrm{mg} / \mathrm{dl}$ para hombres y mujeres, respectivamente ${ }^{16}$. Para colesterol-LDL se estableció como nivel de 
riesgo $>100 \mathrm{mg} / \mathrm{dl}^{16}$. Para los triglicéridos séricos se consideró límite alto $\geq 150 \mathrm{mg} / \mathrm{dl}^{16}$.

d) Glicemia. Se consideró glicemia elevada en ayuno $\geq 100 \mathrm{mg} / \mathrm{dl}^{6}$ y aquellos con valor $\geq 126$ $\mathrm{mg} / \mathrm{dl}$ se consideraron como sospecha de diabetes. Los sujetos que ingerían hipoglicemiantes fueron considerados diabéticos.

e) Sobrepeso y obesidad. Se consideró sobrepeso cuando el Índice de Masa Corporal (IMC =peso/ talla ${ }^{2}$ ) fue entre $\geq 25$ y $<30 \mathrm{~kg} / \mathrm{m}^{2}$ y obesos $\geq 30$ $\mathrm{kg} / \mathrm{m}^{2} 17$. Para los mayores de 65 años se utilizó lo recomendado por el Ministerio de Salud ${ }^{18}$ que clasifica con sobrepeso al IMC entre $\geq 28$ y $<30 \mathrm{~kg} / \mathrm{m}^{2}$ y obeso $\geq 30 \mathrm{~kg} / \mathrm{m}^{2}$.

f) Perímetro de cintura. Se consideró obesidad abdominal al perímetro de cintura $>102 \mathrm{~cm}$ en hombres y $>88 \mathrm{~cm}$ mujeres ${ }^{3}$.

g) Actividad física. Se definió como sedentaria a las personas que durante el último mes no habían practicado deportes o realizado actividad física fuera del horario de trabajo, durante 30 min o más cada vez, al menos 3 veces a la semana ${ }^{6,19}$.

h) Tabaquismo. Se consideró como fumadoras a las personas que declararon fumar uno o más cigarros al día al momento de la entrevista ${ }^{6}$.

i) Coexistencia de FRCV. Para el estudio de coexistencia de FRCV se incluyeron: presión arterial alta, colesterol elevado, DM conocida+glicemia $\geq 126 \mathrm{mg} / \mathrm{dl}$ y tabaquismo.

Este estudio fue aprobado por el Comité de Ética de la Universidad de Talca y el Servicio de Salud del Maule. Además de enviar a domicilio los resultados de las diferentes mediciones, a quienes presentaron alteración(es), se les sugirió consultar a un médico.

Procesamiento y análisis de datos. Los datos fueron ingresados por un digitador en una base de datos ACCESS especialmente diseñada. Previo al análisis de los datos, se realizó la validación y consistencia de la información obtenida. Por otra parte, se calculó la prevalencia y su intervalo de confianza (IC) de 95\% de los FRCV. La presencia/ ausencia de cada FRCV se analizó mediante un modelo de regresión logística ajustando por variables sociodemográficas: sexo, edad, NSE y nivel educacional. A partir de estos modelos se encuentran las razones de ventaja (odds ratio, OR) de padecer el FRCV y sus intervalos de confianza. El análisis se realizó con el software SAS 9.1.3.

\section{RESULTADOS}

Se encuestó a 1.136 personas, de ellas 1.007 $(88,6 \%)$ concurrieron a realizarse mediciones antropométricas, de presión arterial y exámenes de sangre. De éstas, 66,3\% fueron mujeres y 33,7\% hombres (Tabla 1) sin diferencias en la edad, $45,6 \pm 13,5$ y $44,3 \pm 15,1$ años, respectivamente. En su mayoría, la población se encontraba entre los 25 y 64 años, sin diferencias según sexo (Tabla 1). Al estratificar el nivel socioeconómico, los grupos más representados fueron medio-alto, medio y medio bajo, sin diferencias según sexo. Respecto a la educación, 53,1\% de la población tenía entre 8 y 12 años de escolaridad (Tabla 1 ).

Presión arterial aumentada. El 36,7\% (IC 95\% 33,8-39,7) presentó presión arterial aumentada; de ellos 36,5\% desconocía esta condición y el resto reportó tener hipertensión en tratamiento. Esta prevalencia aumentaba significativamente con la edad ( $p<0,0001$ ), alcanzando a $76,9 \%$ en el grupo de 65-74 años, sin diferencias significativas según sexo (Tabla 2), NSE y nivel educacional. La mayor prevalencia en el nivel socioeconómico bajo y nivel educacional básico, se explican porque en esos grupos predominan personas de mayor edad. El grupo de mayor edad tenía 37,2 veces mayor riesgo de presentar presión arterial aumentada que el grupo más joven (IC OR 95\% 14,4-96,4).

Hipercolesterolemia. El 44,5\% (IC 95\% 41,4-47,6) presentó hipercolesterolemia; la prevalencia aumentaba significativamente con la edad ( $p<0,0001$ ), llegando a 63,5\% en el grupo de 65-74 años, sin diferencias significativas por sexo (Tabla 2), NSE y nivel educacional. Este grupo de edad tenía 7,3 veces más riesgo de presentar hipercolesterolemia que el grupo de 18 a 24 años (IC OR 95\% 3,6-14,8). El 39,2\% (IC 95\% 36,2-42,2) presentó colesterol-HDL bajo y 65,3\% presentó colesterol-LDL sobre el nivel deseable (Tabla 3). El 21,9\% presentó colesterol-LDL entre $131-160 \mathrm{mg} / \mathrm{dl}$ y $7,0 \%>160 \mathrm{mg} / \mathrm{dl}$. En la misma tabla se muestra que $40,1 \%$ (IC 95\% 37,1-43,1) de las personas estudiadas presentó hipertrigliceridemia. Sólo 0,5\% presentó sobre $800 \mathrm{mg} / \mathrm{dl}$. 
Tabla 1. Caracterización de la población estudiada por sexo.

\begin{tabular}{|lcrrr|}
\hline Variable & $\begin{array}{c}\text { Total } \\
\mathrm{n}(\%)\end{array}$ & $\begin{array}{c}\text { Hombres } \\
\mathrm{n}(\%)\end{array}$ & $\begin{array}{c}\text { Mujeres } \\
\mathrm{n}(\%)\end{array}$ & Valor p \\
\hline Edad (años) & $73(7,3)$ & $32(9,4)$ & $41(6,1)$ & 0,17 \\
$\quad 18-24$ & $420(41,7)$ & $144(42,5)$ & $276(41,3)$ & \\
$25-44$ & $410(40,7)$ & $126(37,2)$ & $284(42,5)$ & \\
$45-64$ & $104(10,3)$ & $37(10,9)$ & $67(10,0)$ & \\
$\quad 65-74$ & $50(5,0)$ & $17(5,0)$ & $33(4,9)$ & 0,20 \\
Nivel socioeconómico & $353(35,1)$ & $133(37,7)$ & $220(32,9)$ & \\
$\quad$ Muy alto & $288(28,6)$ & $92(27,1)$ & $196(29,3)$ & \\
Alto & $279(27,7)$ & $82(24,2)$ & $197(29,5)$ & \\
$\quad$ Medio alto & $37(3,7)$ & $15(4,4)$ & $22(3,3)$ & \\
Medio bajo & $158(15,7)$ & $40(11,8)$ & $118(17,7)$ & \\
$\quad$ Bajo & $535(53,1)$ & $175(51,6)$ & $360(53,9)$ & \\
Nivel educacional & $314(31,2)$ & $124(36,6)$ & $190(28,4)$ & \\
$\quad<8$ años & $1.007(100)$ & $339(33,7)$ & $668(66,3)$ & \\
8-12 años & & & & \\
$>12$ años & & & & \\
Total & & & & \\
\end{tabular}

Glicemia elevada. El 26,3\% (IC 95\% 41,4-47,6) presentó glicemia elevada en ayuno (Tabla 2). El $6,1 \%$ reportó tener diabetes en tratamiento y 2,1\% tenía sospecha de diabetes. El $21,6 \%$ de la población sin DM conocida tenía glicemia $\geq 100 \mathrm{mg} / \mathrm{dl}$. La prevalencia fue significativamente mayor en las personas de más edad ( $p<0,0001$ ); el grupo de 65 a 74 años tenía 39,4 veces mayor riesgo de presentar hiperglicemia que el grupo más joven (IC OR 95\% 9,2-169,8). Por otra parte, los hombres tenían 1,6 veces mayor riesgo de glicemia aumentada que las mujeres (IC OR 95\% 1,2-2,2). No se encontraron diferencias según NSE y nivel educacional.

Tabaquismo. El 36,5\% (IC 95\% 33,6-39,5) era fumador, siendo significativamente mayor la prevalencia en el grupo de 25 a 44 años ( $p<0,0001)$, sin diferencias según sexo (Tabla 2), NSE y nivel educacional. El grupo de 25-44 años tenía 5,0 veces mayor riesgo de fumar que el grupo de 6574 años (IC OR 95\% 2,8-8,9). Sólo 2,0\% era gran fumador (más de 20 cigarrillos diarios) y 17,1\% era ex-fumador.

Sedentarismo. El 79,9\% (IC 95\% 77,5-82,4) fue considerado sedentario (Tabla 2). En los hombres se observó una tendencia estadísticamente significativa al aumento del sedentarismo con la edad, en cambio en las mujeres la tendencia es a disminuir con la edad (efecto modificador o de interacción) ( $p=0,02)$. No se encontró diferencias según NSE y nivel educacional.

Sobrepeso y obesidad. El 70,1\% (IC 95\% 67,2-72,9) presentó IMC $>25 \mathrm{~kg} / \mathrm{m}^{2}$, distribuidos en $37,5 \%$ que presenta sobrepeso y $32,6 \%$ obesidad (Tabla 2). Se observó un efecto de interacción expresado en que los hombres disminuyeron la prevalencia entre los 65 y 74 años, mientras que las mujeres la mantuvieron ( $\mathrm{p}=0,008$ ). El 3,4\% presentó obesidad mórbida $\left(>40 \mathrm{~kg} / \mathrm{m}^{2}\right)$. Con respecto al nivel educacional, los sujetos con 8 a 12 años de escolaridad tenían 1,7 veces mayor riesgo de sobrepeso-obesidad que los sujetos con más de 12 años de escolaridad (IC 95\% 1,2-2,3).

Perímetro de cintura aumentado. El 41,1\% (IC 95\% 38,1-44,2) de la población estudiada presentó obesidad abdominal. Esta prevalencia aumentó con la edad, existiendo un efecto modificador por sexo, que se expresa en un mayor aumento en las mujeres ( $p<0,0001$ ) (Tabla 2). Los sujetos con menos de 8 años de escolaridad tenían 2,1 veces 
Tabla 2. Prevalencia de factores de riesgo cardiovascular por edad y sexo

\begin{tabular}{|c|c|c|c|}
\hline Factor de riesgo & $\begin{array}{l}\text { Total } \\
\text { n (\%) }\end{array}$ & $\begin{array}{c}\text { Hombres } \\
\text { n (\%) }\end{array}$ & $\begin{array}{c}\text { Mujeres } \\
\text { n (\%) }\end{array}$ \\
\hline \multicolumn{4}{|l|}{ Presión arterial aumentada } \\
\hline $18-24$ años & $6(8,2)$ & $5(15,6)$ & $1(2,4)$ \\
\hline 25-44 años & $85(20,2)$ & $33(22,9)$ & $52(18,8)$ \\
\hline 45-64 años & $199(48,5)$ & $66(52,4)$ & $133(46,8)$ \\
\hline 65-74 años & $80(76,9)$ & $28(75,7)$ & $52(77,6)$ \\
\hline \multicolumn{4}{|l|}{ Hipercolesterolemia } \\
\hline$(>200 \mathrm{mg} / \mathrm{dl})$ & $448(44,5)$ & $137(40,4)$ & $311(46,6)$ \\
\hline 18-24 años & $14(19,2)$ & $6(18,8)$ & $8(19,5)$ \\
\hline 25-44 años & $134(31,9)$ & $49(34,0)$ & $85(30,8)$ \\
\hline 45-64 años & $234(57,1)$ & $59(46,8)$ & $175(61,6)$ \\
\hline 65-74 años & $66(63,5)$ & $23(62,2)$ & $43(64,2)$ \\
\hline \multicolumn{4}{|l|}{ Glicemia aumentada en ayuno } \\
\hline$(\geq 100 \mathrm{mg} / \mathrm{dl})$ & $265(26,3)$ & $105(31,0)$ & $160(24,0)$ \\
\hline 18-24 años & $2(2,7)$ & $1(3,1)$ & $1(2,4)$ \\
\hline 25-44 años & $60(14,3)$ & $27(18,8)$ & $33(12,0)$ \\
\hline 45-64 años & $150(36,6)$ & $58(46,0)$ & $92(32,4)$ \\
\hline 65-74 años & $53(51,0)$ & $19(51,4)$ & $34(50,8)$ \\
\hline Tabaquismo & $368(36,5)$ & $134(39,5)$ & $234(35,0)$ \\
\hline 18-24 años & $30(41,1)$ & $13(40,6)$ & $17(41,5)$ \\
\hline 25-44 años & $192(45,7)$ & $71(49,3)$ & $121(43,8)$ \\
\hline 45-64 años & $131(32,0)$ & $44(34,9)$ & $87(30,6)$ \\
\hline 65-74 años & $15(14,4)$ & $6(16,2)$ & $9(13,4)$ \\
\hline Sedentarismo & $805(79,9)$ & $262(77,3)$ & $543(81,3)$ \\
\hline 18-24 años & $56(76,7)$ & $19(59,4)$ & $37(90,2)$ \\
\hline 25-44 años & $335(79,8)$ & $113(78,5)$ & $222(80,4)$ \\
\hline 45-64 años & $334(81,5)$ & $99(78,6)$ & $235(82,8)$ \\
\hline 65-74 años & $80(76,9)$ & $31(83,8)$ & $49(73,1)$ \\
\hline Sobrepeso y obesidad & $705(70,1)$ & $250(74,0)$ & $455(68,1)$ \\
\hline 18-24 años & $28(38,4)$ & $15(46,9)$ & $13(31,7)$ \\
\hline 25-44 años & $287(68,3)$ & $113(78,5)$ & $174(63,0)$ \\
\hline 45-64 años & $324(79,0)$ & $103(81,7)$ & $221(77,8)$ \\
\hline 65-74 años & $66(64,1)$ & $19(52,8)$ & $47(70,1)$ \\
\hline \multicolumn{4}{|l|}{ Perímetro de cintura aumentado } \\
\hline (hombres $>102 \mathrm{~cm}$, mujeres $>88 \mathrm{~cm}$ ) & $414(41,1)$ & $83(24,5)$ & $331(49,6)$ \\
\hline 18-24 años & $8(11,0)$ & $4(12,5)$ & $4(9,8)$ \\
\hline 25-44 años & $130(31,1)$ & $28(19,4)$ & $102(37,0)$ \\
\hline 45-64 años & $219(53,4)$ & $41(32,5)$ & $178(62,7)$ \\
\hline 65-74 años & $57(54,8)$ & $10(27,0)$ & $47(70,2)$ \\
\hline
\end{tabular}

$\mathrm{n}$ total: 1.007, salvo para sobrepeso y obesidad: 1.006 .

mayor riesgo que los sujetos con más de 12 años de escolaridad de tener el perímetro de cintura aumentado (IC $95 \%$ 1,4-3,3).
Coexistencia de FRCV. Sólo $21,5 \%$ de la población no presentó FRCV, 39,7\% 1, 31,5\% 2, 6,3\% 3 y 1,1\% 4. La coexistencia de FRCV clasifica- 
dos según grupos etarios se muestran en la Figura 1.

\section{DisCUSIÓN}

Es conocido que las ECV son la principal causa de muerte en el mundo ${ }^{1}$ y que en Chile, debido a la transición demográfica que entre otros aspectos se caracteriza por aumento de la esperanza de vida y por mal nutrición en exceso, han aumentado en importancia relativa ${ }^{19}$. En particular la Región del Maule es una de las regiones del país que presenta una tasa de mortalidad cardiovascular por sobre la mediana nacional ${ }^{4}$.

Uno de los objetivos del Programa de Investigación en Factores de Riesgo Cardiovascular (PIFRECV) es caracterizar epidemiológicamente a la

Tabla 3. Prevalencia de dislipidemias por sexo

\begin{tabular}{|c|c|c|c|}
\hline Factor de riesgo & $\begin{array}{l}\text { Total } \\
\text { n }(\%)\end{array}$ & $\begin{array}{c}\text { Hombres } \\
\mathrm{n} \quad(\%)\end{array}$ & $\begin{array}{l}\text { Mujeres } \\
\mathrm{n} \quad(\%)\end{array}$ \\
\hline $\begin{array}{l}\text { Hipercolesterolemia } \\
(>200 \mathrm{mg} / \mathrm{dl})\end{array}$ & $448 \quad(44,5)$ & $137 \quad(40,4)$ & $311 \quad(46,6)$ \\
\hline $\begin{array}{l}\text { Colesterol-LDL aumentado } \\
(>100 \mathrm{mg} / \mathrm{dl})\end{array}$ & $633 \quad(65,3)$ & $216 \quad(67,7)$ & $417 \quad(64,1)$ \\
\hline $\begin{array}{l}\text { Colesterol-HDL disminuido } \\
(\mathrm{H}:<40 \mathrm{mg} / \mathrm{dl}, \mathrm{M}:<50 \mathrm{mg} / \mathrm{dl})\end{array}$ & $395 \quad(39,2)$ & $123 \quad(36,3)$ & $272 \quad(40,7)$ \\
\hline $\begin{array}{l}\text { Hipertrigliceridemia } \\
(\geq 150 \mathrm{mg} / \mathrm{dl})\end{array}$ & $404 \quad(40,1)$ & $162 \quad(47,8)$ & $242 \quad(36,2)$ \\
\hline
\end{tabular}

HDL: lipoproteínas de alta densidad; LDL: lipoproteínas de baja densidad; H: hombres; M: mujeres; $\mathrm{n}$ total: 1.007 , salvo para colesterol-HDL: 970.

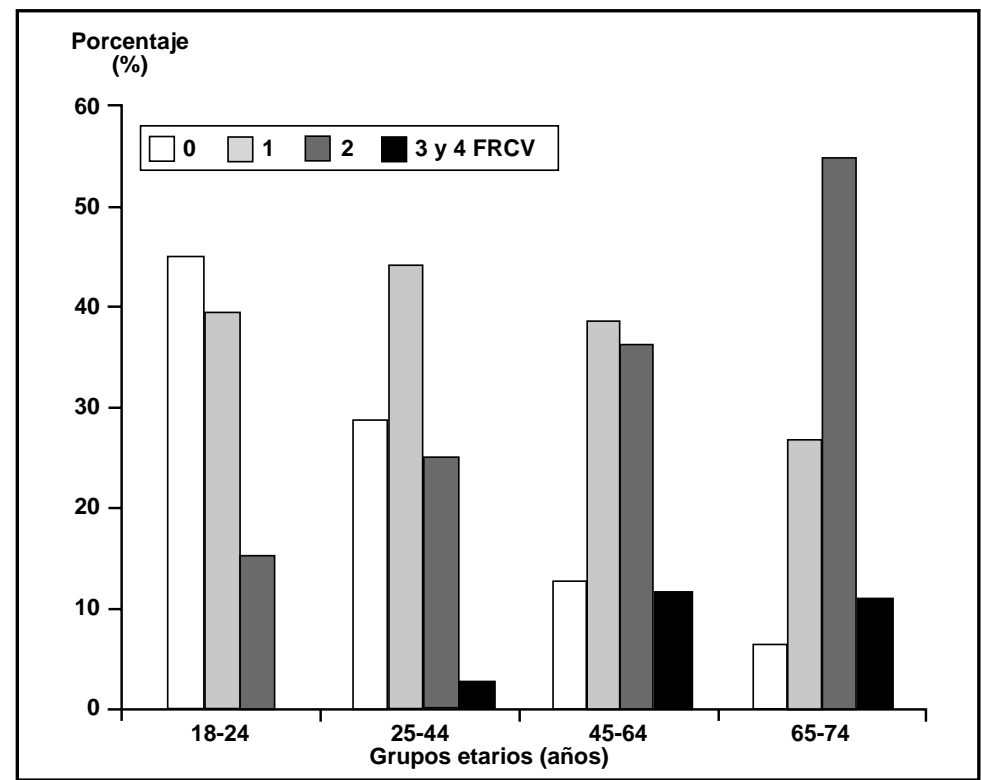

Figura 1. Coexistencia de factores de riesgo cardiovascular por edad. FRCV, factor de riesgo cardiovascular. 
población de la Región del Maule en relación con FRCV. En este contexto realizamos este primer estudio en la ciudad de Talca, donde no existía información basal representativa.

Presión arterial elevada. La prevalencia de presión arterial elevada encontrada en esta población es significativamente superior a 33,7\% encontrado en la $\mathrm{ENS}^{5}$, a $32,9 \%$ hallado por Lanas et $\mathrm{al}^{15}$, a $28,0 \%$ descrito por Fagalde $\mathrm{M}$ et $\mathrm{al}^{21}$ y a $20,9 \%$ encontrado en el programa CARMEN, realizado en Valparaíso ${ }^{22}$. Si se considera el aumento de expectativa de vida de la población, tres cuartas partes de los adultos mayores debieran estar en programas de crónicos bajo control de su presión arterial; además de $8 \%$ de los menores de 25 años.

Dislipidemas. Entre los FRCV mayores la hipercolesterolemia fue el más prevalente. La cifra encontrada es significativamente superior a 35,0\% observado en la ENS $^{6}$ y similar a $46,9 \%$ encontrado en el programa CARMEN ${ }^{22}$. Éste es el factor biológico más prevalente en los jóvenes, similar a lo reportado anteriormente en alumnos de la Universidad de Talca ${ }^{23}$. La frecuencia de hipertrigliceridemia también fue significativamente superior a la encontrada en la ENS (30,0\%).

Glicemia elevada. La prevalencia de glicemia elevada en ayuno en este estudio es una cifra preocupante considerando que esta condición ofrece mayor riesgo de desarrollar diabetes en el futuro ${ }^{24}$. Esta prevalencia incluye a 6,1\% de diabéticos ya conocidos, cifra que podría aumentar durante la etapa de confirmación, en curso, de individuos con glicemias elevadas. En la Región del Maule, Baechler et $\mathrm{al}^{25}$ encontraron que $5,3 \%$ de individuos mayores de 20 años presentó DM, cifra muy similar $(5,1 \%)$ a la encontrada en la ENS para la misma región y para el país $(4,5 \%)^{6}$. El marcado aumento de la prevalencia de hiperglicemia en las personas sobre 25 años refuerza la importancia de incrementar programas de prevención focalizados especialmente antes de la cuarta década.

Tabaquismo. La prevalencia de tabaquismo fue significativamente menor a la encontrada en el programa CARMEN de $40,6 \% 22$ y en la ENS de $42,4 \%{ }^{6}$. A diferencia de ambos estudios, en que se encontró una mayor prevalencia de tabaquismo en hombres, en nuestro estudio no encontramos diferencia según sexo. En Chile recientemente (agosto, 2006) se ha puesto en vigencia la ley antitabaco; será interesante estudiar nuevamente en los próximos años la prevalencia de tabaquismo para evaluar el efecto de la citada ley.

Sedentarismo. La gran mayoría de los encuestados indicaron ser sedentarios. En otros estudios que utilizaron criterios iguales o similares para definir sedentarismo, encontraron prevalencia levemente superior, $80 \%-91 \% 6,15,22$. Es posible que la menor prevalencia se explique porque la encuesta, aplicada en primavera, consultó por la actividad física realizada durante el último mes; en esa época del año un cierto porcentaje de la población deja de ser sedentario.

Sobrepeso y obesidad. La prevalencia encontrada de sobrepeso $\mathrm{u}$ obesidad fue significativamente superior a la encontrada en la ENS $(61,1 \%)^{6}$. Considerando que la obesidad es un factor fuertemente relacionado al estilo de vida, que condiciona no sólo ECV, sino DM y otras patologías, es necesario reforzar programas de prevención.

Perímetro de cintura. La prevalencia de perímetro de cintura aumentado representa una cifra significativamente superior a la encontrada en la ENS $(29,5 \%)^{6}$. Al igual que en dicha encuesta la prevalencia fue significativamente mayor en las mujeres. Si consideráramos los valores más estrictos sugeridos por el reciente consenso europeo (hombres hasta 90 y mujeres hasta $80 \mathrm{~cm}$ ), la prevalencia de esta alteración sería mayor ${ }^{26}$.

Coexistencia de FRCV. La mayoría de la población presentó menos de tres FRCV. Llamó la atención que la presencia de tres o más factores es similar en el grupo de 45-64 y 65-74 años ( $p$ 0,85). Por otra parte, la población sin FRCV disminuye progresivamente con la edad, mientras que aquélla con dos factores aumenta.

El riesgo más alto (tres o cuatro FRCV) se presenta en 7,4\% de la población, cifra similar a la población en riesgo máximo considerada por la presencia de DM.

En el grupo etario de 18-24 años destaca como factor único alterado, el tabaquismo, seguido de hipercolesterolemia y presión arterial alterada. 
En resumen, en la población adulta de la ciudad de Talca encontramos alta prevalencia de la mayoría de los FRCV clásicos, por sobre el porcentaje nacional. Por otra parte, la mayoría de los FRCV aumentan con la edad y algunos muestran diferencia según sexo o nivel educacio-

\section{REFERENCIAS}

1. World Health Organization. Informe sobre la salud en el mundo. Technical Report Series ISBN 924356207 X. Geneve: WHO. 2002.

2. Berríos $X$. Tendencias en factores de riesgo para enfermedades crónicas: zuna nueva epidemia? Rev Méd Chile 1997; 125: 1405-7.

3. Bustos P, Amigo H, Arteaga A, Acosta AM, Rona RJ. Factores de riesgo de enfermedad cardiovascular en adultos jóvenes. Rev Méd Chile 2003; 131: 97380.

4. ICAZA G, NúÑEz L. Atlas de mortalidad cardiovascular en Chile, 1997-2003. Proyecto FONIS SA 04I2005, Financiamiento: CONICYT-MINSAL, 2005. Disponible en http://pifrecv.utalca.cl

5. Keil U, Liese AD, Hense HW, Filitak B, Doring A, StiEBer J ET AL. Classical risk factors and their impact on incident non-fatal and fatal myocardial infarction and all-cause mortality in southern Germany. Results from the MONICA Augsburg cohort study 1984-1992. Monitoring Trends and Determinants in Cardiovascular Diseases. Eur Heart J 1998; 19: 1197-207.

6. Ministerio de Salud de Chile. Encuesta Nacional de Salud 2003. Departamento de Salud Pública de la Pontificia Universidad Católica de Chile. Informe Técnico. 2003

7. Kish L Muestreo de Encuestas. México: Trillas, 1972.

8. НАвітсн J. Estandarización de métodos epidemiológicos cuantitativos sobre el terreno. Bol Sanit Panam 1974; 76: 375-84.

9. Ministerio de Salud, Programa de Salud del AdulTo. Manual: Examen de salud preventivo del adulto». Norma General Técnica №9. MINSAL. 1995.

10. Friedewald W, Levy R, Fredrickson D. Estimation of the concentration of low-density lipoprotein cho- nal. Siendo las ECV la principal causa de morbimortalidad y potencialmente prevenibles tratando los factores de riesgo modificables y sus condicionantes, esperamos que este estudio sea un aporte para mejorar el conocimiento de la epidemiología regional y nacional.

lesterol in plasma, without use of the preparative ultracentrifuge. Clin Chem 1972; 18: 499-502.

11. MÉNDEZ R. El nivel socioeconómico Esomar. VIII Congreso Chileno de Marketing de Icare. 1999.

12. The sixth report of the Joint National Committee on prevention, detection, evaluation, and treatment of high blood pressure. Arch Intern Med 1997; 157: 2413-46.

13. 1999 World Health Organization-International Society of Hypertension Guidelines for the Management of Hypertension. Guidelines Subcommittee. J Hypertens 1999; 17: 151-83.

14. Marchesini G, Forlani G, Cerreli F, Manini $R$, Natale S, Baraldi L et al. WHO and ATPIII proposals for the definition of the metabolic syndrome in patients with type 2 diabetes. Diabet Med 2004; 21: 383-7.

15. Lanas F, Del Solar J, Maldonado M, Guerrero M, Espinoza F. Prevalencia de factores de riesgo de enfermedad cardiovascular en una población de empleados chilenos. Rev Méd Chile 2003; 131: 129-34.

16. Grundy SM, Cleeman Ji, Merz CN, Brewer HB JR, Clark LT, HunninghaKe DB et al. Implications of recent clinical trials for the National Cholesterol Education Program Adult Treatment Panel III guidelines. Circulation 2004; 110: 227-39.

17. US Department of Health and Human Services. Public Health Service. National Institutes of Health. Clinical Guidelines on the Identification, Evaluation and Treatment of Overweight and Obesity in adults. NIH Publication. № 98-4083. 1998.

18. Aibala C, Bunout D, Carrasco F. Evaluación del estado nutricional del senescente. En: Castillo C, Uauy R and Atalah E eds. Manual de Alimentación y Nutrición del Adulto Mayor. MINSAL/ INTA/Depto Nutrición Med Norte. 7-12. 1998. Disponible en http://www.microweb.cl/idm/documentos/ESOMAR.pdf 
19. Berríos $X$, Jadue L, Zenteno J, Ross Mi, Rodríguez $H$. Prevalencia de factores de riesgo para enfermedades crónicas. Estudio en la población general del área metropolitana, 1986-1987. Rev Méd Chile 1990; 118: 597-604.

20. Los objetivos sanitarios de la década 2000-2010. Ministerio de Salud, Gobierno de Chile.

21. Fagalde MDP, Del Solar J, Guerrero M, Atalah E. Factores de riesgo de enfermedades crónicas no transmisibles en funcionarios de una empresa de servicios financieros de la Región Metropolitana. Rev Méd Chile 2005; 133: 919-28.

22. Jadue L, Vega J, Escobar MC, Delgado I, Garrido C, LASTRA P ET AL. Factores de riesgo para las enfermedades no transmisibles: Metodología y resultados globales de la encuesta de base del programa CARMEN (Conjunto de Acciones para la Reducción Multifactorial de las Enfermedades no Transmisibles). Rev Méd Chile 1999; 127: 100413.

23. Palomo I, Torres G, Alarcón M, Maragaño P, Leiva E, Mujica V. Alta prevalencia de factores de riesgo cardiovascular clásicos, en una población de estudiantes universitarios de la región centro-sur de Chile. Rev Esp Cardiol 2006; 59: 1099-105.

24. DekKer J, Balkau B. Counterpoint: impaired fasting glucose: The case against the new American Diabetes Association guidelines. Diabetes Care 2006; 29: 1173-5.

25. Baechler R, Mujica V, Aqueveque X, Ramos L, Soto A. Prevalencias de Diabetes Mellitus en la Séptima Región de Chile. Rev Méd Chile 2002; 130: 1257-64.

26. Zimmet P, Maglano D, Matsuzana Y, Alberti G, SHaw J. The metabolic syndrome: a global public health problem and a new definition. J Atheroscler Thromb 2005; 12: 295-300.

\section{Agradecimientos}

Agradecemos a la Dirección de Investigación de la Universidad de Talca por el financiamiento del Programa de Investigación de Factores de Riesgo de Enfermedades Cardiovasculares (PIFRECV) y a la empresa Roche Laboratories, Mannheim, Alemania por la donación de los reactivos para determinar perfil lipídico y glicemia de este estudio. 